\title{
EXPOSICIÓN Y RIESGO OCUPACIONAL DE COVID-19 EN ESTUDIANTES, DOCENTES Y PROFESIONALES DE LA SALUD
}

\author{
Narváez Olalla Alberto ${ }^{1}$, Melena Zapata Jonathan ${ }^{2 *}$, Guerrero González Jhon ${ }^{1}$, Solís Cárdenas Diana ${ }^{1}$, \\ Calderón Layedra Lilian ${ }^{1}$, Albán Villacis Jorge ${ }^{1}$, Piedra Andrade Jefferson ${ }^{3}$, Padilla Contreras Rocío'. \\ DOI: 10.48018/rmv.v31.i2.5
}

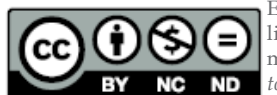

Esté bajo una licencia de Creative Commons de tipo ReconocimienOPEN ACCESS derivadas 4 - International.

1. Universidad Central del Ecuador. Facultad de Ciencias Médicas. Carrera de Medicina. Quito - Ecuador. 2. Gestión de la Seguridad Clínica del Paciente y Calidad de la Atención Sanitaria. Quito - Ecuador. 3. Hospital de Especialidades Carlos Andrade Marín. Médico en Funciones Hospitalarias. Quito - Ecuador.

ORCID ID:

Narváez Olalla Alberto

orcid.org/0000-0002-5056-6315.

Melena Zapata Jonathan

orcid.org/0000-0003-2212-5130

Guerrero González Jhon

orcid.org/0000-0002-0797-8103

Solís Cárdenas Diana

orcid.org/0000-0003-2278-4435

Calderón Layedra Lilian

orcid.org/0000-0003-4788-2621

Albán Villacis Jorge

orcid.org/0000-0002-3549-7454

Piedra Andrade Jefferson

orcid.org/0000-0003-3003-065X

Padilla Contreras Rocío

orcid.org/0000-0003-1072-9404

*Corresponding author: Melena Zapata Jonathan E-mail: jonathanmelena94@gmail.com

Article history

Received: 26 - Jun - 2020

Accepted: 27 - Sep - 2020

Publish: 01 - Oct - 2020

STROBE 2008 Check List statement: The authors have real the STROBE 2008 Check List and the manuscript was prepared and revised according to the STROBE 2008 Checklist.

Conflict of interest: All authors declared that there are no conflicts of interest.

Financial disclosure: The authors have no financial relationships relevant to this article to disclose.

\section{Authors' contribution:}

AN y JM: concepción del estudio, recolección de datos, análisis e interpretación de datos, redacción del artículo. JG, DS y LC: recolección de datos, análisis e interpretación de datos y la redacción del artículo.

JA, JP y RP: recolección, análisis e interpretación de datos.

All the authors reviewed and approved the final manuscript.

Citation: Narváez Olalla A, Melena Zapata J, Guerrero González J, Solís Cárdenas D, Calderón Layedra L, Albán Villacis J, et al. EXPOSICIÓN Y RIESGO OCUPACIONAL DE COVID-19 EN ESTUDIANTES, DOCENTES Y PROFESIONALES DE LA SALUD. Rev Med Vozandes. 2020; 31 (2) $33-41$

\section{Resumen}

\section{Introducción}

La exposición de los profesionales de la salud al nuevo coronavirus (SARS-CoV-2) así como el riesgo de adquirir su enfermedad asociada (COVID-19) es más alta en comparación a otros grupos poblacionales. La correcta implementación de medidas de bioseguridad puede reducir su riesgo de infección. El objetivod e este estudio fue evaluar la disponibilidad de insumos y Equipos Personales de Protección (EPP), las exigencias y riesgos ocupacionales y su relación con el COVID-19.

\section{Pacientes y métodos}

Estudio observacional descriptivo de corte transversal que incluyó a 603 participantes entre estudiantes, docentes de una universidad pública y profesionales de la salud. Las mediciones principales fueron: exposición ocupacional e incidencia de COVID-19.

\section{Resultados}

El 73,5\% de encuestados fueron mujeres, el $92,6 \%$ adultos jóvenes y el 23,55\% (IC95\% 20,3-27, 1) refirió diagnóstico de COVID-19 (15\% confirmado, $8 \%$ sospechoso). Laborar en la provincia de Pichincha y no disponer de jabón se asoció con diagnóstico de COVID-19 (OR ajustado= 2.85 y 2.68 respectivamente). El contacto con casos confirmados y sospechosos fueron las variables que presentaron asociaciones más fuertes con la enfermedad (OR ajustado= 9.28 y 3.07 respectivamente).

\section{Conclusiones}

La alta incidencia de COVID-19 en los encuestados se asoció con deficiencias en la disponibilidad de EPP. La protección de profesionales de salud debe ser una prioridad para las autoridades de salud y trabajo, quienes además deben brindar los EPP e insumos necesarios. Recomendamos el tamizaje periódico de este grupo ocupacional para evaluar el impacto de las medidas de protección y analizar la implementación de correcciones necesarias.

Palabras clave: Infecciones por Coronavirus, Coronavirus, Exposición Profesional, Equipo de Protección Personal, Evaluación de Riesgo para la Salud. 
Keywords: Coronavirus Infections, Coronavirus, Occupational Exposure, Personal Protective Equipment, Health Risk Assessment.

\section{Abstract \\ EXPOSURE AND OCCUPATIONAL RISK OF COVID-19 IN STUDENTS, TEACHERS AND HEALTH PROFESSIONALS.}

\begin{abstract}
Introduction
Health professional's exposure to the new coronavirus (SARS-CoV-2) as well as their risk of acquiring COVID-19 "its associated disease", has been higher compared to other population groups. Nevertheless, the correct implementation of biosecurity measures could reduce their infection risk. The objective of this study was to evaluate the availability of personal protective equipment "PPE", occupational risks and its relationship with COVID-19 in health professionals.
\end{abstract}

\author{
Patients and methods \\ 603 subjects among students, teachers, and health professionals were included in a cross- \\ sectional descriptive observational study. Occupational exposure and incidence of COVID-19 \\ were the main measurements.
}

\begin{abstract}
Results
Most of the subjects were women $(73.5 \%)$ and young adults $192.6 \%)$ and $23.55 \%(95 \% \mathrm{Cl} 20.3$ 27.1) referred a diagnosis of COVID-19 (15\% confirmed, $8 \%$ suspected). People who worked in Pichincha's province and those who did not have soap presented a higher risk of COVID-19 infection (adjusted $\mathrm{OR}=2.85$ and 2.68 respectively). Contact with confirmed and suspicious cases were the variables that were associated with the highest risk of infection (adjusted OR= 9.28 and 3.07 respectively).

\section{Conclusions}

The high incidence of COVID-19 in the subjects was associated with PPE deficiencies. Health professional's protection must be a priority for health and labor authorities, who must also provide the PPE and necessary supplies. A periodic screening in this occupational group to assess the impact of protective measures and analyze the implementation of necessary corrections.is recommended.
\end{abstract}

\section{Introducción}

A fines de diciembre de 2019, se produjo un brote del síndrome respiratorio agudo severo causado por el nuevo coronavirus (SARS-CoV-2) que produce la enfermedad por coronavirus 2019 (COVID-19) en Wuhan, provincia de Hubei-China, desde donde se extendió a más de 210 países. Actualmente, todos los continentes se encuentran afectados con excepción de la Antártida (1).

A pesar de que varios países europeos y asiáticos han iniciado un proceso gradual de desescalada. En América, actual epicentro de la pandemia, los contagios crecen de forma sostenida. Los países más afectados son los Estados Unidos de Norteamérica (EUA) con más de 3,7 millones de contagios y 140.000 fallecimientos, seguido de Brasil, Perú y México (2).

Una gran proporción de casos reportados de COVID-19 fueron relacionados con la exposición ocupacional, inicialmente a comerciantes y compradores de mercados de Wuhan. Sin embargo, con la expansión de la pandemia, los Profesionales de la Salud (PS) fueron reconocidos como otro grupo de alto riesgo para la infección por SARS-COV-2 ${ }^{(3)}$

Para el 22 de enero en Wuhan ya se reportaron 15 PS infectados por COVID-19. En un estudio publicado en China el 7 de febrero, reportó que el $29 \%$ de casos confirmados de COVID-19 correspondió a PS ${ }^{(4)}$. Se ha observado que el riesgo de infección para SARS-CoV-2 depende del tiempo de exposición y contacto con personas infectadas, así como con la carga viral adquirida ${ }^{(5)}$.

La revisión sistemática realizada por Jefferson et al en el año 2011, las deficiencias en la disponibilidad de Equipos de Protección Personal 
(EPP) en PS se relacionaron con mayor riesgo de transmisión de infecciones respiratorias virales.

La ausencia de respiradores N95, guantes y gafas se fueron las variables que se asociaron con el mayor riesgo de transmisión (6).

En el Reino Unido, la British Medical Association (BMA, en inglés) advirtió que, a pesar de las garantías ofrecidas por el gobierno respecto a la provisión de suministros médicos, el PS aún se encuentran en "riesgo considerable" debido a la escasez de EPP. Motivo por el cual el 30 de marzo la BMA mencionó que busca una claridad del gobierno sobre los riesgos que el personal de los hospitales y los médicos generales de atención primaria deberían o no asumir en caso de la carencia de EPP (7).

En Ecuador, medios de comunicación locales reportaron quejas del personal del sistema sanitario sobre falta de insumos médicos para su protección. Hasta el 3 de mayo se reportaron 20937 casos confirmados de COVID-19, de los cuales el 16,5\% (3462) correspondieron a PS (8).

Según Ortiz et al, al inicio de la pandemia de COVID-19 en Ecuador (febrero a abril), el $78 \%$ de todos los casos confirmados $(9,468)$ correspondieron a mestizos, el 19\% (1800) fueron PS, de los cuáles los médicos (876) fueron el grupo más afectados al representar el 9,3\% del total de casos notificados. El sexo masculino, la edad avanzada, la presencia de comorbilidades, trabajar como obrero o estar desempleado se asoció con mayor riesgo de muerte por COVID-19 ${ }^{(9)}$. Lo anterior destaca la importancia de evaluar los riesgos y exposiciones laborales de PS en torno al COVID-19(10).

El objetivo del estudio fue evaluar la disponibilidad de insumos y EPP, las exigencias y riesgos ocupacionales y su relación con el COVID-19 en estudiantes, docentes de una universidad pública y profesionales de la salud para sugerir recomendaciones de prevención de la enfermedad.

\section{Pacientes y Métodos}

\section{Diseño de estudio}

Estudio observacional descriptivo de corte transversal.

\section{Desarrollo de la Encuesta}

Se aplicó una encuesta en línea a través de la herramienta Google Forms y se publicó por redes sociales (Facebook y WhatsApp) y correos electrónicos de estudiantes internos rotativos y posgradistas, docentes y PS egresados de la Facultad de Ciencias Médicas de la Universidad Central del Ecuador (FCM-UCE). El período de recolección de datos fue desde el 24 hasta el 30 de marzo del 2020, un mes de después de la detección del primer caso de COVID-19 en Ecuador(11).

A partir del formulario de Google Forms se construyó una base de datos en el programa Microsoft Excel.

\section{Participantes}

Se incluyó 603 participantes que correspondieron a 413 estudiantes de internado rotativo de medicina (IRM), 110 estudiantes de posgrado de medicina, 51 docentes y 29 PS egresados de la FCM-UCE. Más de la mitad de estudiantes de postgrado fueron de la especialidad Medicina Familiar y Comunitaria debido a que la suspensión de actividades administrativas impidió el acceso a correos electrónicos y redes sociales de estudiantes de postgrados diferentes.

\section{Variables}

Esta encuesta forma parte del sistema de vigilancia de COVID-19 específico para estudiantes, trabajadores, docentes y egresados de la FCM-UCE que se realizará cada seis meses. Las preguntas fueron elaboradas en base a los objetivos del estudio y la revisión bibliográfica realizada por los autores.

El instrumento se validó a través de una prueba piloto en 15 estudiantes de la FCM-UCE cinco días previos a la aplicación de las encuestas. Se validó la comprensión de las preguntas y las alternativas de respuesta.

La encuesta fue anónima y autoadministrada. Se solicitó consentimiento informado al inicio de la encuesta.

Se recopiló información sobre características sociodemográficas, (edad, sexo, cargo, carrera, lugar y nivel de atención de la institución donde labora), exigencias ocupacionales, percepción de riesgo, disponibilidad de insumos para desinfección de manos (jabón, toallas, alcohol gel) y EPP (mascarilla, mandil, gafas, guantes, gorros, terno completo) y capacitación sobre COVID-19. Se preguntó la condición diagnóstica de COVID-19 de cada participante y se unieron las alternativas confirmadas y sospechosos como variable dependiente.

La pregunta sobre contacto con casos sospechosos y confirmados fue añadida después de que respondieran a la encuesta, por lo que solo 446 (74\%) de los 603 respondieron a esta pregunta. Respecto a la disponibilidad de EPP se construyó un puntaje asignando el valor 1 a los que disponían y 0 a los que no disponían.

\section{Sesgos}

Debido a las características del instrumento de recolección de datos, no se tuvo control sobre sesgos de selección ni tampoco se comprobó los resultados de las pruebas de confirmación diagnóstica que al momento de la encuesta eran Reacción en Cadena de la Polimerasa con Transcriptasa Inversa (PCR-RT). Además, por el limitado acceso a pruebas PCR se incluyeron a personas con diagnóstico sospechoso de COVID-19, lo cual pudo incluir a falsos positivos.

\section{Tamaño muestral}

Se estimó el tamaño de muestra mínimo con el programa STATCALC de Epi Info 7.2. con poder: 80\%, Razón de Momios: 2 y porcentaje de casos 
expuestos: 35\%. El tamaño mínimo de muestra fue de 400. Con el objetivo de mejorar la precisión del estudio se mantuvo la encuesta hasta completar 603 respuestas.

\section{Análisis estadístico}

A partir de un formulario en Google Forms se construyó una base de datos en el programa Excel. El análisis univariado y bivariado se realizó con el programa Epi Info 7.2. Variables continuas se expresaron como promedios y desviación estándar y variables cualitativas en porcentajes. La comparación entre grupos fue realizada usando el Test T de Student's o el Test de MannWhitney para variables continuas. Variables categóricas fueron comparadas usando el Test de Chi-cuadrado de Pearson o Test exacto de Fisher como apropiado. Variables con valor $P<0,2$ fueron incluidas en el modelo multivariado mediante regresión logística binaria utilizando el paquete estadístico IBM SPSS software package, versión 23.0 for Windows (IBM, Armonk, NY, USA). Se utilizó el cut off de 0,2 al ser la significación estadística más baja aceptada (12)

\section{Aspectos Éticos}

El instrumento fue aprobado por el directorio de la Comisión de Salud Pública de la FCM- UCE y expuesto a las autoridades de la UCE. Debido al carácter anónimo de la encuesta los nombres e identificación de los participantes no fueron incluidos, los resultados obtenidos fueron estandarizados y codificados para asegurar su confidencialidad y se siguió las directrices STROBE para estudios observacionales ${ }^{(13)}$.

\section{Resultados}

\section{Características sociodemográficas}

Participaron en la encuesta 603 personas. Hubo un predominio de mujeres $(73,47 \%)$ y adultos jóvenes $(92,64 \%)$. La media de la edad fue 32 años ( \pm 5.8$)$. El $68,49 \%$ de participantes fueron estudiantes de IRM, el 18,24\% estudiantes de postgrado de medicina, el $8,46 \%$ docentes y el $4,81 \%$ PS.

\section{Condiciones laborales}

El $92 \%$ de encuestados trabajaba en establecimientos de la provincia de Pichincha. El $42,12 \%$ de establecimientos fueron de segundo nivel de atención, el 38,14\% de tercero y cuarto y el $19 \%$ de primero. La mayoría de encuestados trabajaba en la red de salud pública: Ministerio de Salud Pública (MSP): 56,2\% e Instituto Ecuatoriano de Seguridad Social (IESS): 34,5\%; sólo el 1,33\% en la red privada. Uno de cada diez participantes refirió padecer alguna enfermedad crónica mientras el 5\% refirió administración de medicamentos inmunosupresores. El $47 \%$ de entrevistados no recibieron capacitación sobre COVID-19 y en el $13 \%$ de casos no se modificó la jornada laboral

Disponibilidad EPP e insumos de desinfección de manos

La mayoría de participantes refirió no disponer

\begin{tabular}{|c|c|c|c|c|c|c|}
\hline \multirow[t]{2}{*}{$\begin{array}{l}\text { Variables } \\
(n=603)\end{array}$} & \multicolumn{2}{|c|}{$\begin{array}{l}\text { Confirmados \& sospechosos } \\
\qquad(n=142)\end{array}$} & \multicolumn{2}{|c|}{$\begin{array}{l}\text { No infectados } \\
(n=461)\end{array}$} & \multirow[t]{2}{*}{ OR (IC95) } & \multirow[t]{2}{*}{ Valor $p$} \\
\hline & $\mathbf{N}$ & $\%$ & $\mathbf{N}$ & $\%$ & & \\
\hline Sexo (Hombre) & 41 & 25.63 & 119 & 74.38 & $1.16(0.76-1.78)$ & 0.26 \\
\hline Edad Media (DE) & 29.97 & $(5.80)$ & 27.89 & (8.29) & DM: 2.08 (0.61-3.55) & 0.005 \\
\hline Primer nivel de Atención & 44 & 36.97 & 75 & 63.03 & $2.31(1.49-3.56)$ & $<0.0001$ \\
\hline \multicolumn{7}{|l|}{ Cargo } \\
\hline Residentes posgrado & 63 & 57.27 & 47 & 47.73 & Chi-2=90.95 & $<0.0001$ \\
\hline Docentes/ Profesionales & 18 & 22.50 & 62 & 77.50 & & \\
\hline Internos rotativos & 61 & 14.77 & 352 & 85.23 & & \\
\hline \multicolumn{7}{|l|}{ Provincia de trabajo } \\
\hline Pichincha & 124 & 22.67 & 423 & 77.33 & Chi-2= 2.95 & 0.4 \\
\hline Imbabura & 6 & 37.50 & 10 & 62.50 & & \\
\hline Tungurahua & 3 & 27.27 & 8 & 72.73 & & \\
\hline Otras & 9 & 31.03 & 20 & 68.97 & & \\
\hline \multicolumn{7}{|l|}{ Institución } \\
\hline IESS & 43 & 20.67 & 165 & 79.33 & Chi-2= 3.28 & 0.51 \\
\hline MSP & 84 & 24.78 & 255 & 75.22 & & \\
\hline ISFA & 12 & 32.35 & 23 & 67.65 & & \\
\hline ISPOL & 3 & 21.43 & 11 & 78.57 & & \\
\hline Privada & 1 & 12.50 & 7 & 87.50 & & \\
\hline
\end{tabular}


de ternos $(97,51 \%)$, gafas $(86,9 \%)$, mandiles $(77,61 \%)$ o gorros (69\%). Sin embargo, más de la mitad refirió disponer de guantes $(60,3 \%)$ y mascarillas $(77,2 \%)$. El promedio de EPP disponibles por cada entrevistado fue bajo $2,16( \pm 1,34)$ de un total de seis equipos.

A pesar que la mayoría de participantes refirió disponer de insumos para la desinfección de manos: gel desinfectante $(89,7 \%)$, jabón $(90,5 \%)$ y toalla $(76,12 \%)$, apenas el $14,8 \%$ refirió un acceso permanente a EPP e insumos de desinfección de manos. En el 18,7\% de casos la provisión de EPP e insumos siempre fue realizada por la institución, en el 52,2\% la provisión fue ocasional.

\section{Incidencia COVID-19}

El $36.10 \%$ de encuestados refirieron contacto con casos sospechosos y el 5,83\% con casos confirmados. El 15,42\% (IC95\% 12,76-18,52) de encuestados refirieron diagnóstico confirmado de COVID-19 y el $8.13 \%$ fueron sospechosos (IC95\% $6,20-10,58)$. Al considerar los casos confirmados y sospechosos, la incidencia del COVID-19 fue de 23,55\% (IC95\% 20,3-27,1).

Según los grupos poblaciones observados, la incidencia más alta de COVID-19 se observó en estudiantes de posgrado de medicina $(44,37 \%)$ y de IRM $(42,96 \%)$. En docentes y PS la incidencia fue considerablemente menor $(10,56 \%$ y $2,11 \%)$.

\section{Exposición laboral al COVID-19}

La mayoría de encuestados no trabajó en servicios de alta exposición a enfermos COVID-19 como triaje, aislamiento, unidad de terapia intensiva (UTI) o transporte de pacientes COVID-19. Sin embargo, la mayor exposición se observó en los servicios de triaje donde uno de cada diez encuestados trabajó de forma permanente y el $23,4 \%$ lo hizo en rotaciones.

En la mayoría de casos la jornada laboral y el horario de trabajo fueron modificados a turnos de 24 horas cada tercer, cuarto o quinto día. Los internos rotativos y los residentes de posgrado trabajaron más de 160 horas al mes.

Se observó una diferencia de medias entre infectados y no infectados estadísticamente significativa (DM 2,08 IC=0,61-3,55) $p=0,005$. Los encuestados que trabajaban en establecimientos de primer nivel de atención ( $O R=2,31 ; p<0,0001)$ y los residentes de posgrado presentaron mayor tamaño de efecto de infección (Chi-2=90,95; $p<0,0001$ ); tabla 1.

\begin{tabular}{|c|c|c|c|c|c|c|}
\hline \multirow[t]{2}{*}{ Variables $(n=603)$} & \multicolumn{2}{|c|}{$\begin{array}{c}\text { Confirmados \& } \\
\text { sospechosos }(n=142)\end{array}$} & \multicolumn{2}{|c|}{ No infectados $(n=461)$} & \multirow[t]{2}{*}{ OR (IC95\%) } & \multirow[t]{2}{*}{ Valor $p$} \\
\hline & $\mathbf{N}$ & $\%$ & $\mathbf{N}$ & $\%$ & & \\
\hline \multicolumn{7}{|l|}{ Exposiciones } \\
\hline Con contacto sospechoso & 42 & 26.09 & 119 & 73.91 & $4.43(2.51-7.82)$ & $<0.0001$ \\
\hline Con contacto confirmado & 16 & 61.54 & 10 & 38.46 & $12.69(5.44-29.60)$ & $<0.0001$ \\
\hline En UTI & 12 & 20,69 & 46 & 79,31 & $0.83(0.43-1.62)$ & 0.36 \\
\hline En servicios de aislamiento & 34 & 28,10 & 87 & 71,90 & $1.35(0.86-2.12)$ & 0,11 \\
\hline En servicios de triaje & 68 & 34,34 & 130 & 65,66 & $2.34(3.44-1.59)$ & $<0.0001$ \\
\hline En servicios de transporte & 32 & 25,81 & 92 & 74,19 & $1.17(0.74-1.84)$ & 0.39 \\
\hline \multicolumn{7}{|l|}{ Condiciones de riesgo } \\
\hline Tener enfermedad Crónica & 19 & 30.16 & 44 & 69.84 & $1.46(0.82-2.60)$ & 0.20 \\
\hline Tomar medicamento inmunosupresor & 11 & 36.67 & 19 & 63.33 & $1.95(0.90-4.20)$ & 0.11 \\
\hline \multicolumn{7}{|l|}{ Percepción de Riesgo } \\
\hline Muy alto & 9 & 42.86 & 12 & 57.14 & Chi-2= 13.22 & 0.0102 \\
\hline Alto & 23 & 21.30 & 85 & 78.70 & & \\
\hline Moderado & 49 & 19.60 & 201 & 80.40 & & \\
\hline Bajo & 16 & 15.69 & 86 & 84.31 & & \\
\hline Muy bajo & 5 & 8.47 & 54 & 91.53 & & \\
\hline \multicolumn{7}{|l|}{ Exigencias laborales } \\
\hline No modificación de jornada & 24 & 28.92 & 59 & 71.08 & $1.38(0.82-2.32)$ & 0.21 \\
\hline Ausencia capacitación & 64 & 22.38 & 222 & 77.62 & $0.88(0.60-1.28)$ & 0.56 \\
\hline Horas trabajo mes (Media DE) & 158,81 & 16,72 & 157,2 & 19,07 & $\begin{array}{c}\text { DM: } 1.61(-1.9 \mathrm{a} \\
-5.12)\end{array}$ & 0.45 \\
\hline
\end{tabular}

OR: Odds Ratios, DM: Diferencia de Medias, DE: Desviación Estándar, UTI: Unidad de Terapia Intensiva, Nivel de significancia: valor de $\mathrm{p}<0.05$. 
Tabla 3. Relación entre disposición de equipos de protección e insumos para desinfección de manos con infección por COVID-19. Ecuador, 2020.

\begin{tabular}{|c|c|c|c|c|c|c|}
\hline \multirow[t]{2}{*}{ Variables $(n=603)$} & \multicolumn{2}{|c|}{$\begin{array}{l}\text { Confirmados \& sos- } \\
\text { pechosos }(n=142)\end{array}$} & \multicolumn{2}{|c|}{ No infectados $(n=461)$} & \multirow[t]{2}{*}{ OR (IC95\%) } & \multirow[t]{2}{*}{ Valor $p$} \\
\hline & $\mathrm{N}$ & $\%$ & $N$ & $\%$ & & \\
\hline \multicolumn{7}{|l|}{ EPP } \\
\hline No disponer de mascarilla & 12 & 15.58 & 65 & 84.42 & $0.56(0.29-1.07)$ & 0.08 \\
\hline No disponer de mandil & 108 & 23.08 & 360 & 76.92 & $0.89(0.57-1.38)$ & 0.64 \\
\hline No disponer de gafas & 123 & 23.47 & 401 & 76.53 & $0.96(0.55-1.68)$ & 0.88 \\
\hline No disponer de guantes & 61 & 25.52 & 178 & 74.48 & $1.19(0.81-1.75)$ & 0.37 \\
\hline No disponer de gorros & 104 & 25.00 & 312 & 75.00 & $1.30(0.85-1.98)$ & 0.25 \\
\hline No disponer de terno & 139 & 23.64 & 449 & 76.36 & $1.23(0.34-4.45)$ & 1 \\
\hline Núm. EPP: Media (DE) & 3.45 & $(2.20)$ & 3.81 & $(2.17)$ & DM: $-0.36(-0.7$ a -0.0$)$ & 0.08 \\
\hline \multicolumn{7}{|c|}{ Insumos desinfección de manos } \\
\hline No disponer de jabón & 27 & 47.37 & 30 & 52.63 & $3.37(1.92-5.90)$ & $<0.0001$ \\
\hline No disponer de toalla & 53 & 36.81 & 91 & 63.19 & $2.42(1.60-3.64)$ & $<0.0001$ \\
\hline No disponer de gel alcohol & 18 & 29.03 & 44 & 70.97 & $1.37(0.76-2.46)$ & 0.27 \\
\hline \multicolumn{7}{|c|}{$\begin{array}{l}\text { Disponibilidad de EPP e insumos } \\
\text { desinfección de manos }\end{array}$} \\
\hline Nunca & 9 & 18.75 & 39 & 81.25 & Chi-2= 2.777 & 0.2494 \\
\hline A veces & 117 & 25.11 & 349 & 74.89 & & \\
\hline Siempre & 16 & 17.98 & 73 & 82.02 & & \\
\hline \multicolumn{7}{|c|}{$\begin{array}{l}\text { Institución provee de EPP e insu- } \\
\text { mos desinfección de manos }\end{array}$} \\
\hline Nunca & 40 & 22.86 & 135 & 77.14 & Chi-2= 3.273 & 0.1947 \\
\hline A veces & 82 & 26.03 & 233 & 73.97 & & \\
\hline Siempre & 20 & 17.70 & 93 & 82.30 & & \\
\hline \multicolumn{7}{|c|}{$\begin{array}{l}\text { Encuestado lleva EPP e insumos } \\
\text { desinfección de manos }\end{array}$} \\
\hline Nunca & 39 & 23.64 & 126 & 76.36 & Chi-2=0.7011 & 0.7043 \\
\hline A veces & 79 & 24.53 & 243 & 75.47 & & \\
\hline \multicolumn{7}{|l|}{ Siempre } \\
\hline & 24 & 20.69 & 92 & 79.31 & & \\
\hline
\end{tabular}

OR: Odds Ratios, DM: Diferencia de Medias, DE: Desviación Estándar, EPP: Equipos de protección personal, Nivel de significancia: valor de $\mathrm{p}<0.05$.

En relación a la exposición, condiciones de riesgo y exigencias laborales la presencia de enfermedades crónicas, la administración de medicamentos inmunosupresores y la percepción de riesgo muy alta mostraron asociaciones positivas estadísticamente significativas con el diagnóstico de COVID-19. No se encontraron diferencias significativas con las variables (modificación de la jornada y horas de trabajo); tabla 2.

Respecto a los EPP e insumos de desinfección, el no disponer de jabón o toalla se asoció de forma positiva con diagnóstico de COVID-19 $(p<0,0001)$, el resto de variables no mostraron asociaciones significativas; tabla 3.
En el análisis multivariado el contacto con casos confirmados y sospechosos de COVID-19 fueron las variables que presentaron asociaciones más fuertes con el diagnóstico de COVID-19 (OR ajustado $=9,28$ y 3,07 respectivamente). Laborar en establecimientos de salud de la provincia de Pichincha y no disponer de jabón para desinfección de manos también se asociaron con el diagnóstico de COVID-19 (OR ajustado= 2,85 y 2,68 respectivamente); tabla 4. 
Tabla 4. Incidencia de COVID-19 en entrevistados según características demográficas, factores de riesgo y disponibilidad de EPP e insumos. Ecuador, 2020.

\begin{tabular}{|c|c|c|c|c|c|c|c|}
\hline \multirow[t]{2}{*}{ Variables } & B & Error estándar & Wald & Sig. & $\operatorname{Exp}(B)$ & $95 \%$ C.I. para $\operatorname{Exp}(B)$ & \\
\hline & & & & & & Inferior & Superior \\
\hline Provincia Pichincha & 1.049 & .403 & 6.768 & .009 & 2.856 & 1.295 & 6.296 \\
\hline Contacto sospechosos & 1.122 & .304 & 13.647 & .000 & 3.070 & 1.693 & 5.565 \\
\hline Contactos confirmados & 2.228 & .457 & 23.732 & .000 & 9.286 & 3.788 & 22.761 \\
\hline No disponer de jabón & .989 & .441 & 5.032 & .025 & 2.688 & 1.133 & 6.375 \\
\hline Constante & -2.778 & .244 & 130.046 & .000 & .062 & & \\
\hline
\end{tabular}

Fuente: Elaborado por los autores

\section{Discusión}

La incidencia acumulada del COVID-19 en los encuestados durante el 24 al 30 de marzo, período correspondiente al inicio de la epidemia y del aislamiento nacional, fue del 23\%. Por el carácter anónimo de la encuesta, no se pudo investigar el resultado de los casos sospechosos, lo que puede determinar una subestimación de la incidencia. En informes oficiales del 29 de mayo se reportaron 3054 PS infectados, la mayoría fueron médicos (1.672), enfermeras (788) y auxiliares de enfermería (381) ${ }^{(14)}$.

Los estudiantes de posgrado y de IRM presentaron las incidencias más altas de COVID-19 (44\% y 42\% respectivamente). En el caso de residentes de posgrado, el contacto directo con pacientes en servicios con alto riesgo de exposición viral, la elevada transmisibilidad del SARS-CoV-2 a través de aerosoles y el elevado porcentaje de asintomáticos pueden explicar dicho hallazgo ${ }^{(15)}$.

En el presente estudio la incidencia del COVID-19 en estudiantes de IRM fue elevada, por lo cual se firmó un Memorándum de Entendimiento entre la FCM- UCE, la Zona 9 del MSP y el Fondo de las Naciones Unidas para la Infancia (UNICEF) para garantizar la disponibilidad de EPP a estudiantes de IRM (16).

En Ecuador al 30 de marzo del 2020, fecha en la que la encuesta fue concluida, 1966 casos confirmados fueron reportados en 23 provincias. Las más afectadas según el número de casos confirmados fueron: Guayas con 1397, Pichincha 191, Los Ríos 60, Azuay 57 y Manabí 50. La incidencia observada en el presente estudio fue mayor a la reportada a nivel provincial y nacional(17)

El trabajar en la provincia de Pichincha se asoció de forma significativa con el diagnóstico del COVID-19. Este hallazgo se puede explicar por un sesgo de selección, porque la mayoría de participantes trabajaban en Pichincha, provincia que al momento de la encuesta ocupaba el segundo lugar según el número de casos reportados. Mientras que en las otras provincias (Tungurahua, Imbabura y Carchi), donde trabajaban otros residentes de postgrado e internos rotativos, los casos reportados eran escasos ${ }^{(17)}$.

La edad avanzada y la presencia de comorbilidades pueden elevar el riesgo de adquirir el COVID-19 (18) (19). En el presente estudio dicha asociación no fue observada, probablemente porque la mayoría de encuestados fueron menores de 50 años. Tampoco se observó que el sexo o el lugar de trabajo influyan en el riesgo de infección.

La disponibilidad de EPP no se asoció con el diagnóstico del COVID-19, sin embargo, el elevado número de encuestados que carecían de EPP es un hallazgo importante. El gobierno ecuatoriano emitió el 28 de abril del 2020 a través de la Resolución No. MDT-2020-022 del Ministerio del Trabajo que "la enfermedad del coronavirus (COVID-19) no constituye un accidente de trabajo ni una enfermedad profesional", y posteriormente, el 29 de abril de 2020 modificó el art.l de dicha resolución y añadió la excepción de aquellos casos en los que se pudiera establecer de forma científica o por métodos adecuados a las condiciones y a las prácticas nacionales, un vínculo directo entre la exposición a agentes biológicos que resulte de las actividades laborales contraídas por el trabajador ${ }^{(20)}$.

La no disponibilidad de jabón entre los encuestados se asoció con mayor frecuencia de infección por el SARS-CoV-2. El bajo número de participantes que disponían permanentemente de insumos de desinfección contrasta con lo sugerido por la evidencia científica que indica que el uso de agua y jabón es el método más eficiente para la desinfección de manos ${ }^{(21,22)}$.

La higiene adecuada de manos es una de las herramientas más efectivas para prevenir infecciones asociadas a la asistencia sanitaria, infecciones cruzadas y propagación de resistencia antimicrobiana. Sin embargo, el cumplimiento de esta medida se ve afectado por la disponibilidad de insumos, el conocimiento de la técnica adecuada, los factores humanos, el medio ambiente y el liderazgo efectivo. La pandemia del COVID-19 ha enfatizado la importancia de mantener la práctica efectiva de higiene de manos durante todo momento ${ }^{(23)}$. 
En este estudio la disponibilidad del gel a base de alcohol no se asoció con la enfermedad. Sin embargo, es un método efectivo para la higienización de manos antes y después del contacto directo con pacientes y durante la atención clínica, excepto en situaciones en las que se debe usar agua y jabón (21). A pesar que su uso puede reducir la propagación de infecciones por bacterias, su efectividad contra algunos virus no envueltos como rotavirus y norovirus es reducida y depende de la concentración de alcohol(24). Por lo cual, en casos de exposición viral confirmada o sospechosa, se recomienda el uso de gel a base de alcohol como medida de seguridad adicional(25).

El promedio de EPP (menor a 4 sobre 6 ) refleja el incumplimiento del Artículo 16- Convenio sobre seguridad y salud de los trabajadores, 1981 (núm. 155) de la Organización Internacional del Trabajo (OIT) en la que establece que el empleador tiene deberes en relación con el suministro y la utilización de EPP en el lugar de trabajo. El cual además establece que un EPP es un equipo que protege al usuario del riesgo de accidentes o de efectos adversos para la salud. En el caso de PS el equipo depende del nivel de exposición y riesgo ${ }^{(26)}$. El uso correcto de EPP puede reducir el riesgo de infección de enfermedades altamente contagiosas, sin embargo, su cumplimiento puede conllevar mayor dificultad e incomodidad en su empleo y conducir a un efecto contraproducente ${ }^{(27)}$. En el presente estudio dicha relación no fue evaluada.

La sociabilización de protocolos y guías, el apoyo de directivos, la cultura del lugar del trabajo, el espacio físico, el acceso a EPP y la confianza de cada profesional también pueden influir en el uso correcto de EPP (28).

El contacto con casos confirmados y sospechosos fueron las variables que se asociaron con mayor frecuencia de infección por SARS-CoV-2. La elevada transmisibilidad viral y el contacto con líquidos orgánicos contaminados de pacientes puede explicar el mayor riesgo de infección del PS durante epidemias de enfermedades altamente infecciosas como las producidas por el virus del Ébola (EVE) o el Síndrome Respiratorio Agudo Grave (en inglés, SARS). Las precauciones de contacto mediante el uso de EPP pueden reducir su riesgo (29).

La realización de pruebas diagnósticas exhaustivas, la vigilancia y el aislamiento del PS potencialmente expuesto al SARS-CoV-2 puede no ser factible en sistemas de salud colapsados. Sin embargo, la vigilancia del personal es un componente crucial del control de brotes durante la fase de contención epidémica para reducir la probabilidad de transmisión nosocomial y mantener la confianza y moral en el sistema de salud (30,31).

Una estrategia centrada en la vigilancia de síntomas de infecciones respiratorias altas para la detección temprana de casos, el manejo de brotes y el cumplimiento a nivel individual contribuye a detectar y contener con éxito la transmisión del SARS-CoV-2 entre PS durante brotes continuos. Además, debido a que la mayoría de casos del COVID-19 son leves y la transmisión local en curso, la vigilancia debe centrarse en la propagación intrahospitalaria en áreas donde los PS pueden tener mayor riesgo de exposición (32).

\section{Limitaciones}

La estimación de la incidencia acumulada del COVID-19 en base a la suma de casos confirmados y sospechosos, así como la ausencia de confirmación diagnóstica debido al formato digital de la encuesta, pudo relacionarse con una sobreestimación de la misma.

El elevado porcentaje de residentes de postgrado de la especialidad de Medicina Familiar y Comunitaria (superior al 50\%) pudo relacionarse a un sesgo de selección y determinar una ausencia de asociación entre el nivel de atención y el riesgo de infección por COVID-19, debido que a sus actividades académicas y asistenciales son realizadas predominantemente en establecimientos de primer nivel de atención. La mayor frecuencia del COVID-19 en la Provincia de Pichincha, puede también deberse a un sesgo de selección porque la mayoría de encuestados trabajaban en esta provincia y el número de encuestados de otras provincias era pequeño. Por lo tanto, la asociación de mayor riesgo de infección de COVID-19 y falta de EPP en participantes de dicha provincia debe interpretarse con prudencia.

Es posible que también exista un sesgo de autoexclusión de profesionales y estudiantes que no percibían estar en riesgo de infección o no presentaban ninguna sintomatología. Lo anterior explicaría una probable sobre estimación de la incidencia de infectados. Sin embargo, no cambia el hecho de que los entrevistados hayan carecido de EPP y de insumos de desinfección de manos, lo cual elevó su riesgo de infección. Dentro de los sesgos de medición de exposición se puede incluir el de recuerdo, sin embargo, su influencia puede ser pequeña. El tamaño de encuestados no ligados a la universidad fue muy pequeño, es posible que en este grupo la incidencia y el riesgo de enfermar sea más alto al observado por el presente estudio.

\section{Conclusión}

Este estudio demuestra que la falta de provisión de EPP e insumos de desinfección de manos, necesarios para garantizar las normas básicas de bioseguridad, eleva el riesgo de transmisión de COVID-19 en PS. Con base a los hallazgos anteriores se recomienda que la protección del PS sea una alta prioridad por parte de las autoridades de salud y del trabajo. Además, debido al elevado número de asintomáticos que no reportan síntomas, el acceso a pruebas de laboratorio a grupos poblacionales prioritarios deber estar garantizado ${ }^{(33)}$. Una nueva encuesta a nivel nacional puede revelar la situación actual de los PS y evaluar la necesidad de implementar nuevas medidas preventivas. 


\section{Referencias}

1. Velavan TP, Meyer CG. The COVID-19 epidemic. Trop Med Int Health. 2020;25(3):278-80.

2. RTVE.es. El mapa mundial del coronavirus: más de 30,6 millones de casos y más de 954.000 muertos en todo el mundo [Internet]. 954.000 muertos en todo el mundo [Internet].
RTVE.es. 2020 [citado 19 de septiembre de 2020]. Disponible en: https://www.rtve.es/ noticias/20200919/mapa-mundial-del-coronavirus/1998143.shtml

3. Koh D. Occupational risks for COVID-19 infection. Occup Med. 12 de marzo de 2020;70(1):3-5.

4. Min L, Peng $H$, Huiguo L, Xiaojiang $W$, Fajiu $L$ Shi $C$, et al. Clinical characteristics of 30 medical workers infected with new coronavirus pneumonia. Chin J Tuberc Respir Dis. 12 de marzo de 2020;43(03):209-14.

5. Wang D, Hu B, Hu C, Zhu F, Liu X, Zhang J, ef al. Clinical Characteristics of 138 Hospitalized Patients With 2019 Novel Coronavirus-Infected Pneumonia in Wuhan, China. JAMA. 17 de marzo de 2020;323(11):1061-9.

6. Jefferson $T$, Mar CBD, Dooley L, Ferroni $E$ Al $\square$ Ansary LA, Bawazeer GA, et al. Physical interventions to interrupt or reduce the sal interventions to interrupt or reduce the spread of respiratory viruses. Cochrane Da-
tabase Syst Rev [Internet]. 2011 [citado 19 de septiembre de 2020];(7). Disponible en: https://www.cochranelibrary.com/cdsr/ doi/10.1002/14651858.CD006207.pub4/full/es

7. Iacobucci G. Covid-19: Doctors still at "considerable risk" from lack of PPE, BMA warns. BMJ [Internet]. 31 de marzo de 2020 [citado 19 de septiembre de 2020];368. Disponible en: https://www.bmj.com/content/368/bmj. $\mathrm{m} 1316$

8. ediciónmédica. Médicos e internos rotativos de Guayaquil denuncian falta de equipos de protección para enfrentar el CoVID19 [Internet]. edicionmedica.ec. 2020 [citado 19 de net]. edicionmedica.ec. 2020 [citado 19 de
septiembre de 2020]. Disponible en: https:// septiembre de 2020]. Disponible en: https://
www.edicionmedica.ec/secciones/profewww.edicionmedica.ec/secciones/profe
sionales/medicos-e-internos-rotativos-de guayaquil-denuncian-falta-de-equipos-deproteccion-para-enfrentar-el-covid 19-95502

9. Ortiz-Prado E, Simbana-Rivera K, Diaz AM Barreto A, Moyano C, Arcos V, et al. Epidemiological, socio-demographic and clinica features of the early phase of the COVID-19 epidemic in Ecuador. medRxiv. 18 de mayo de 2020;2020.05.08.20095943.

10. Li Q, Guan X, Wu P, Wang X, Zhou L, Tong $Y$, et al. Early Transmission Dynamics in WUhan China of Novel Coronavirus-Infected Pneumonia. N Engl J Med [Internet]. 29 de Pneumonia. N Engl J Med [Internet]. 29 de
enero de 2020 [citado 19 de septiembre de 2020]; Disponible en: https://www.nejm.org/ doi/10.1056/NEJMoa2001316

11. Secretaría General de Comunicación de la Presidencia. Se registra el primer caso de coronavirus en Ecuador - Secretaría General de Comunicación de la Presidencia [Internet].

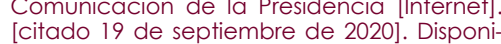
ble en: https://www.comunicacion.gob.ec/ se-registra-el-primer-caso-de-coronavirus-en ecuador/

12. Kim J, Bang $\mathrm{H}$. Three common misuses of $\mathrm{P}$ values. Dent Hypotheses. 2016;7(3):73-80.
13. von Elm E, Altman DG, Egger M, Pocock SJ, Gøtzsche PC, Vandenbroucke JP, et al. The Strengthening the Reporting of Observational Studies in Epidemiology (STROBE) Statement: Guidelines for Reporting Observational Studies. Epidemiology. noviembre de 2007; 18(6):800-804

14. Ministerio de Salud Pública. Boletin NO29 Covid-2019 Ecuador [Internet]. Ministerio de Salud Pública. 2020 [citado 19 de septiembre de 2020]. Disponible en: https://www.salud. gob.ec/wp-content/uploads/2020/03/Boletin-029-PM.pdf

15. CDC. How COVID-19 Spreads [Internet]. Centers for Disease Control and Preven tion. 2020 [citado 20 de septiembre de 2020]. Disponible en: https://www.cdc.gov/ coronavirus/2019-ncov/prevent-getting-sick/ how-covid-spreads.html

16. Universidad Central del Ecuador. BOLETíN DE PRENSA No 246-Firma de Memorando de Entendimiento entre la Universidad Central del Ecuador, Zona 9 de Salud del Ministerio de Salud Pública, Fondo de las Nacione Unidas para la Infancia [Internet]. Universidad Central del Ecuador. 2020 [citado 19 de septiembre de 2020]. Disponible en: http:// repositorio.uce.edu.ec/archivos/jmsalazara/ Boletines/Boletines2020/300/246.pdf

17. Servicio deGestión de Riesgos y Emergen cias. Situación Nacional por COVID-19 (COronavirus) Infografía N033 [Internet]. Servicio deGestión de Riesgos y Emergencias. [citado 19 de septiembre de 2020]. Disponible en: https://www.gestionderiesgos.gob.ec/ wp-content/uploads/2020/03/INFOGRAwp-content/uploads/2020/03/INFOGRA-
FIA-NACIONALCOVI-19-COE-NACIONALFIA-NACIONALCOVI-
30032020-17h00-v3.pdf

18. CDC. Las personas con ciertas afeccione [Internet]. Centers for Disease Control and Prevention. 2020 [citado 19 de septiembre de 2020]. Disponible en: https://espanol.cdc. gov/coronavirus/2019-ncov/need-extra-pregov/coronavirus/2019-ncov/need-extra-precaution

19. CDC. Personas con mayor riesgo de enfermarse gravemente | Coronavirus | COVID-19 I CDC [Internet]. Centro para el Control y la Prevención de Enfermedades. 2020 [citado 4 de junio de 2020]. p. 1-2. Disponible en: https://espanol.cdc.gov/coronavirus/2019 ncov/need-extra-precautions/groups-at-higncov/need-ext
her-risk.html

20. Ministerio del Trabajo. RESOLUCIÓN Nro. MDT-2020-022 [Internet]. Ministerio del Trabajo. 2020 [citado 19 de septiembre de 2020] Disponible en: http://www.trabajo.gob.ec/ wp-content/uploads/downloads/2020/04/ wP-content/upload

21. Loveday HP, Wilson JA, Pratt RJ, Golsorkh $M$, Tingle A, Bak A, et al. epic3: Nationa Evidence-Based Guidelines for Preventing Healthcare-Associated Infections in NHS Hospitals in England. J Hosp Infect. 1 de enero de 2014;86:S1-70.

22. Burton M, Cobb E, Donachie P, Judah G, Curtis V, Schmidt W-P. The Effect of Handwashing with Water or Soap on Bacterial Contamination of Hands. Int J Environ Res Public Health. enero de 2011;8(1):97-104.
23. Hillier MD. Using effective hand hygiene practice to prevent and control infection. Nurs Stand. 27 de abril de 2020;35(5):45-50.

24. Kampf G. Efficacy of ethanol against viruses in hand disinfection. J Hosp Infect. 1 de abril de 2018;98(4):331-8.

25. CDC. Hand Hygiene Recommendations [Infernet]. Centers for Disease Control and Prevention. 2020 [citado 19 de septiembre de 2020]. Disponible en: https://www.cdc.gov/ coronavirus/2019-ncov/hcp/hand-hygiene. $\mathrm{html}$

26. Organización Internacional del Trabajo. C155 - Convenio sobre seguridad y salud de los trabajadores, 1981 (núm. 155) [Internet]. Organización Internacional del Trabajo. 1983 [citado 19 de septiembre de 2020]. Disponible en: https://www.ilo.org/dyn/normlex/es/f? $p=$ NORMLEXPUB:12100:0::NO::P12100 ILO CODE:C 155

27. Verbeek JH, ljaz S, Mischke C, Ruotsalainen $J H$, Mäkelä E, Neuvonen K, et al. Personal protective equipment for preventing highly infectious diseases due to exposure to contaminated body fluids in healthcare staff. Cochrane Database Syst Rev [Internet]. 2016 [citado 19 de septiembre de 2020];(4). Disponible en: https://www.cochranelibrary.com/ cdsr/doi/10.1002/14651858.CD01 1621.pub2/ full

28. Houghton $C$, Meskell $P$, Delaney $H$, Smalle $M$, Glenton C, Booth A, et al. Barriers and facilitators to healthcare workers' adherence with infection prevention and control (IPC) guidelines for respiratory infectious diseases: a rapid qualitative evidence synthesis. Cochrane Database Syst Rev [Internet]. 2020 [citado 19 de septiembre de 2020];(4). Disponible en: https://www.cochranelibrary.com/cdsr/ doi/10.1002/14651858.CD013582/full/es

29. Bero L, Lasserson T Opiyo N, Featherstone R, Mehta M. Coronavirus (COVID-19): medidas de prevención y control de la infección [Internet]. Cochrane. 2020 [citado 19 de septiembre de 2020]. Disponible en: /es/coronavirus-covid-19-medidasde-prevenci\%C3\%B3n-y-control-de-lainfecci\%C3\%B3n

30. Aghaizu A, Elam G, Ncube F, Thomson G Szilágyi $E$, Eckmanns $T$, et al. Preventing the next "SSARS» - European healthcare workers attitudes towards monitoring their health for the surveillance of newly emerging infections: qualitative study. BMC Public Health. 8 de julio de $2011 ; 11$ (1):541

31. Ives J, Greenfield S, Parry JM, Draper H, Gratus C, Petts Jl, et al. Healthcare workers' attitudes to working during pandemic influenza: a qualitative study. BMC Public Health. 12 de febrero de 2009;9(1):56.

32. Wee LE, Sim XYJ, Conceicao EP, Aung MK Goh JQ, Yeo DWT, et al. Containment of COVID-19 cases among healthcare workers: The role of surveillance, early detection, and outbreak management. Infect Control Hosp Epidemiol. julio de 2020;41 (7):765-71.

33. Schrezenmeier E, Dörner T. Mechanisms of action of hydroxychloroquine and chloroquine: implications for rheumatology. Nat Rev Rheumatol. marzo de 2020;16(3):155-66. 